\title{
IV-FMC: an automated vision based part modeling and reconstruction system for flexible manufacturing cells
}

\begin{abstract}
The use of computer vision system in manufacturing industry can eliminate the visual faults due to the limitation of human vision and increase productivity. The aim of the current study is to develop an automated vision system (IV-FMC) to reconstruct manufacturing parts in three-dimensional (3D) model. In the designed system, laser stripes are projected onto an object to be scanned. A charge-coupled device (CCD) camera captures the two dimensional (2D) image from the reflected stripes. Based of the principle of optical triangulation, the distance between the object and the camera is calculated in which the third dimension of the image is obtained. These processes iterate each time the object is rotated in different angles, letting the system to capture the whole view of the object being scanned. A 3D model of the object is then reconstructed by merging multiple range images obtained from the range scanning. A PC-based data acquisition board is designed to control the switching of the laser module. The reconstruction process is automated to form a single $3 \mathrm{D}$ surface model of the object being scanned.
\end{abstract}

Keyword: Automated vision system; Optical triangulation; Reconstruction; Threedimensional (3D) model 